\title{
Simulation Algorithm of Naval Vessel Radiation Noise
}

\author{
XIE Jun \\ Naval Submarine Academy \\ QingDao,China \\ xiejun688@tom.com
}

\author{
DA Liang-long \\ Naval Submarine Academy \\ QingDao,China \\ dalianglong@sohu.com
}

\begin{abstract}
Propeller cavitation noise continuous spectrum results from noise radiated from random collapses of a large number of cavitation bubbles. In consideration of randomicity of initial collapse of cavitation bubbles and similarity of frequency spectrums of various cavitation bubble radiated noises, theoretical analysis is conducted on four types of cavitation radiated noise continuous spectrum characteristics, which shows that simulation modeling could be carried out for cavitation noise continuous spectrum waveform by use of Monte Carlo method based on statistics of cavitation bubble radius and initial collapse time. The model parameters are expected values, difference degree and randomicity of cavitation bubble radius, which will be respectively used to determine base waveform of radiated noise, similarity of various cavitation bubble noise frequency spectrums and random collapse process of a larger number of cavitation bubbles. The input parameters have specific physical meaning and are controlled simply and could simulate the characteristics of cavitation noise continuous spectrum in a better way and therefore the simulation results could be better matching with theoretical analysis.
\end{abstract}

Keywords-waveform simulation; continuous spectrum; propeller cavitation noise; broadband noise

\section{INTRODUCTION}

Propeller cavitation noise is one of important radiated noise source for recognition of underwater targets which has been studied widely by scholars at home and abroad[1-4]. Power spectrum of propeller cavitation noise consists of low frequency line spectrum and continuous spectrum. Such continuous spectrum is recognized to be formed by noises radiated from random collapses of a large number of cavitation bubbles. Statistics of test data show that the continuous spectrum could increase at the rate of 6$12 \mathrm{~dB} /$ octave in case of being lower than peak value and could decrease at the rate of close to $6 \mathrm{~dB} /$ octave in case of being higher than peak value if such continuous spectrum has a peak value in some frequency[5]. For this, frequency domain method will be usually used for broadband cavitation noise continuous spectrum waveform simulation, e.g. use white noise to excite the filter with a given power spectrum shapes so as to acquire broadband cavitation noise waveform sequence with a given continuous spectrum shape[6-8]. The continuous spectrum shapes could be determined by empirical formula such as Ross formula[5] or by parameter model such as Esc model[9-10].

Based on research results of predecessors, time averaging treatment is made in this paper to the time variation of continuous spectrum caused by periodic time variation of stern wake field that is caused by rotation of propellers, namely, time domain statistic simulation is made for propeller broadband cavitation noise waveform by use of Monte Carlo method while taking into account the expected value of continuous spectrum of propeller cavitation radiated noise only. The simulation results show that the input parameters have specific physical meaning and could be controlled simply and could simulate the characteristics of cavitation noise continuous spectrum in a better way.

\section{MODEL FOR NOISE RADIATED FROM SIMULTANEOUS} COLLAPSE OF A LARGE NUMBER OF CAVITATION BUBBLES

Assumption 1: when ship is sailing under given working conditions and sea conditions, the expected value of collapse radius within stern propeller area is $R_{0}$ which is constant.

Assumption 2: individual cavitation bubble collapse radius of cavitation bubble group is random variable $R$, compliance expected value is Rayleigh Distribution of $R_{0}$ and radiated sound pressure pulse is $p_{R}(t)$ and corresponding frequency spectrum is $P_{R}(f)$.

Assumption 3: by neglecting interaction of cavitation bubbles, the far broadband cavitation noise field radius is random variable $R$ which results from noises radiated from cavitation bubble random collapse.

Probability density distribution of cavitation bubble collapse radius $R$ could be inferred from assumption 2:

$$
F(R)=\frac{\pi R}{2 R_{0}{ }^{2}} e^{-\frac{\pi R^{2}}{4 R_{0}{ }^{2}}}, R \geq 0
$$

According to Rayleigh individual cavitation bubble model[5]:

$$
f_{R}=\frac{1}{0.915 R} \sqrt{\frac{P_{\infty}}{\rho}}
$$

In the formula above, $f_{R}$ is cavitation bubble bounce frequency, $P_{\infty}$ is middle and far field pressure of liquid, $\rho$ is liquid density; so, probability density distribution of $f_{R}$ is: 


$$
F\left(f_{R}\right)=\frac{\pi f_{R_{0}}^{2}}{2 f_{R}^{3}} e^{-\frac{\pi f_{R_{0}}^{2}}{4 f_{R}^{2}}}, f_{R} \geq 0
$$

Expected value of cavitation radius is $R_{0}$, power spectrum of broadband synthetic noise radiated from simultaneous collapse of a large number of cavitation bubbles compliant with Rayleigh Distribution.

$$
\begin{aligned}
& |S(f)|^{2}=\left|\int_{0}^{\infty} F\left(f_{R}\right) P_{R}(f) d f_{R}\right|^{2} \\
& =\left|\int_{0}^{\infty} \frac{\pi f_{R_{0}}^{2}}{2 f_{R}^{3}} e^{-\frac{\pi f_{R_{0}}^{2}}{4 f_{R}^{2}}} P_{R}(f) d f_{R}\right|^{2}
\end{aligned}
$$

In formula (4), $S(f)$ is broadband synthetic noise frequency spectrum.

\section{A. The same structure of various cavitation bubble waveforms}

Because the sound pressure pulse peak value is positively proportional to $R^{3 / 2}$, in case of only relative amplitude to be considered, it is as follows:

$$
P_{R}(f)=R^{3 / 2} P_{1}(f)
$$

In formula (5), $P_{1}(f)$ represents cavitation bubble frequency spectrum when $\mathrm{R}=1$, hereinafter called base frequency spectrum. The corresponding power spectrum is called base power spectrum and the corresponding waveform is called base waveform.

It could be worked out as follows from formula (2):

$$
R=\frac{1}{0.915 f_{R}} \sqrt{\frac{P_{\infty}}{\rho}}
$$

Infer with formula (5) and (4):

$$
\begin{aligned}
& |S(f)|^{2}=1.3\left(\frac{P_{\infty}}{\rho}\right)^{3 / 2}\left|\int_{0}^{\infty} \frac{\pi f_{R_{0}}^{2}}{2 f_{R}^{9 / 2}} e^{-\frac{\pi f_{0}^{2}}{4 f_{R}^{2}}} d f_{R}\right|^{2}\left|P_{1}(f)\right|^{2} \quad \text { (7) } \\
& \text { Set } \quad C=1.3\left(\frac{P_{\infty}}{\rho}\right)^{3 / 2}\left|\int_{0}^{\infty} \frac{\pi f_{R_{0}}^{2}}{2 f_{R}^{9 / 2}} e^{-\frac{\pi f_{R_{0}}^{2}}{4 f_{R}^{2}}} d f_{R}\right|^{2} \quad \text {, } \quad \text { is }
\end{aligned}
$$
constant, and it is worked out as follows:

$$
|S(f)|^{2}=C\left|P_{1}(f)\right|^{2}
$$

The analysis process above shows that the broadband synthetic noise power spectrum radiated from simultaneous collapse of a large number of cavitation bubbles of different radius will be exactly the same to base power spectrum in terms of shapes in case amplitude normalized frequency spectrums of various cavitation bubbles radiated noise are the same. Constant $C$ represents proportional coefficient of total noise energy radiated from cavitation bubbles of different radius to radiated energy of each unit of cavitation bubbles. Fig $1 \mathrm{~b}$ is result of simulation analysis which is exactly the same to theoretical analysis above.

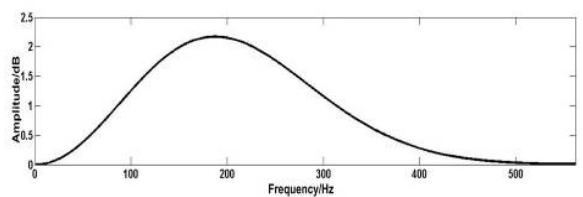

a. Base power spectrum

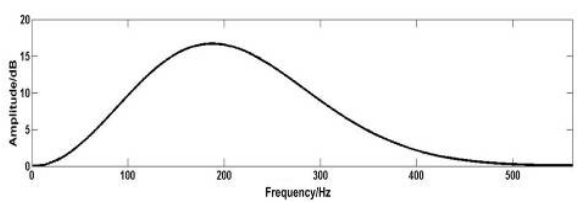

b. Power spectrum of synthetic noise

Figure 1. Simulation results of simultaneous collapse under the same waveform structure

\section{B. Different structures of various cavitation bubble waveform}

From formula (2), the cavitation bubbles collapse time is in positive proportion to cavitation bubbles radius in the same area of liquid; so, the cavitation bubbles of different radius will have different collapse times which will then result in differences between various cavitation bubble radiated noise spectrums.

It is worked out from formula (2) and (4):

$$
|S(f)|^{2}=\left|\int_{0}^{\infty} \frac{\pi R}{2 R_{0}^{2}} e^{-\frac{\pi R^{2}}{4 R_{0}^{2}}} P_{R}(f) d R\right|^{2}
$$

The formula above shows that the broadband synthetic noise power spectrum radiated from simultaneous collapse of cavitation bubbles of different radius is modulus square of weighted sum of noise spectrum radiated from cavitation bubbles of different radius. The time scale factor is used in simulation to describe the effect of time length of cavitation bubbles collapse on the variation of waveform. Therefore, variance $\delta$ of time scale factor can be used to describe the difference of noise spectrum $P_{R}(f)$ radiated from cavitation bubbles of different radius which is called degree of difference. The larger the degree of difference is, the smaller the similarity of broadband synthetic noise power spectrum and base power spectrum is, or vice versa. Fig 2 is simulation result that is worked out from formula (9). The digits in the fig are cavitation bubble radius. $\mathrm{a}$ and $\mathrm{b}$ are simulation results in case of $\delta$ being smaller, and c and d are simulation results in case of $\delta$ being larger. The simulation results verify the correctness of theoretical analysis above. Meanwhile, formula (9) shows that the larger the cavitation bubble radius $R$ is, the larger the radiated noise pressure weight coefficient and the larger the contribution to total sound field is if $R_{0}$ is given. The larger the $R_{0}$ is, the smaller radiated sound pressure weight coefficient is and the smaller the contribution to the total sound field is. 


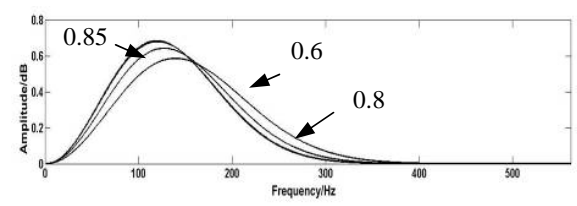

a.Power spectrum of noise radiated from cavitation bubbles of different

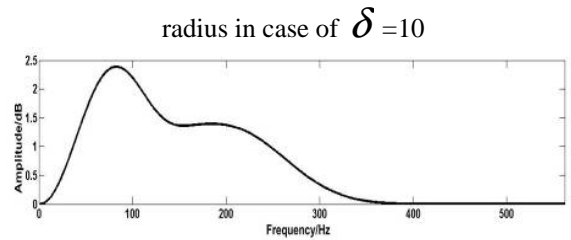

b.Power spectrum of synthetic noise in case of $\delta=10$

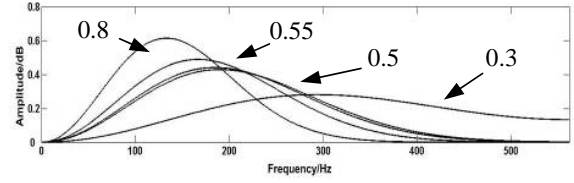

c. Power spectrum of noise radiated from cavitation bubbles of different radius in case of $\boldsymbol{\delta}=50$

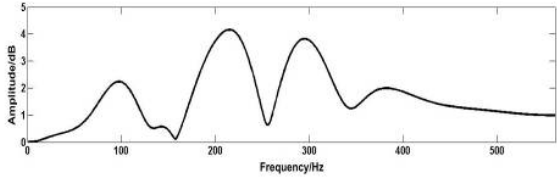

d.Power spectrum of synthetic noise in case of $\boldsymbol{\delta}=50$

Figure 2. Simulation results for various degrees of difference

Two kinds of analysis are conducted on broadband synthetic noise radiated from a large number of cavitation bubbles that are collapsing at the same time. In consideration of random time of collapse of cavitation bubbles, the following analysis is made to the noise radiated from random collapse of a large number of cavitation bubbles.

III. MODEL FOR NOISE RADIATED FROM RANDOM COLLAPSE OF A LARGE NUMBER OF CAVITATION BUBBLES

A. The same structure of various cavitation bubble waveforms

Assume:

$$
p_{R}(t)=R^{3 / 2} p_{1}\left(t-t_{0}\right)
$$

In formula (10), $p_{1}(t)$ is base waveform and $p_{R}(t)$ is single caivation bubble waveform with radius of $\mathrm{R}, t_{0}$ is delay. It can be worked out as follows if Fourier Transform is made to both sides of formula (10):

$$
\begin{aligned}
& \hbar\left\{p_{R}(t)\right\}=R^{3 / 2} \hbar\left\{p_{1}(t)\right\} e^{-j \omega t_{0}} \\
& =R^{3 / 2} P_{1}(f) e^{-j \omega t_{0}}
\end{aligned}
$$

In formula (11), $\hbar$ is operator of Fourier Transform, and angle frequency $\omega=2 \pi f, f$ is frequency and $P_{1}(f)$ is base frequency spectrum; therefore, the spectrum of noise radiated from cavitation bubbles that collapse randomly in a large number and at different times can be expressed as:

$$
\begin{aligned}
& |S(f)|^{2}=\left|\int_{0}^{\infty} \frac{\pi R}{2 R_{0}^{2}} e^{-\frac{\pi R^{2}}{4 R_{0}^{2}}} \int_{-\infty}^{\infty} P_{1}(f) e^{-j \omega t_{0}} d t_{0} d R\right|^{2} \\
& =\left|\int_{0}^{\infty} \frac{\pi R}{2 R_{0}^{2}} e^{-\frac{\pi R^{2}}{4 R_{0}^{2}}} d R \int_{-\infty}^{\infty} P_{1}(f) e^{-j \omega t_{0}} d t_{0}\right|^{2} \\
& =C_{1}\left|\int_{-\infty}^{\infty} P_{1}(f) e^{-j \omega t_{0}} d t_{0}\right|^{2}
\end{aligned}
$$

In formula (12), constant $C_{1}=\int_{0}^{\infty} \frac{\pi R}{2 R_{0}^{2}} e^{-\frac{\pi R^{2}}{4 R_{0}^{2}}} d R$.

It is known from formula (12) that the cavitation bubbles that are different in radius and identical in waveform structure have energy contributions to the broadband synthetic noise power spectrum but will not change shape structure of power spectrum. The change intensity of shape structure of power spectrum depends on $t_{0}$ randomicity and is described by use of variance $\xi$ of $t_{0}$. $\xi$ has more effect on smoothness of synthetic power spectrum and less effect on envelope shape of power spectrum. Fig 3 is simulation results that are based on base waveform and different degrees of randomicity $\xi$.
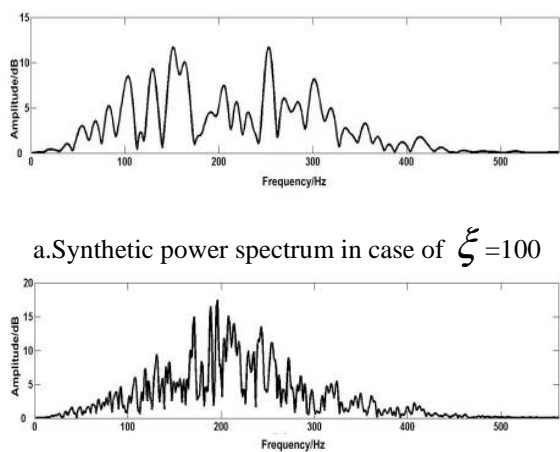

$$
\text { b.Synthetic power spectrum in case of } \xi=400
$$

Figure 3. Simulation results under different degrees of randomicity

\section{B. Different structures of various cavitation bubble waveforms}

It is known from the analysis above that broadband synthetic noise power spectrum radiated from collapse of cavitation bubbles of different radius can be expressed in the following formula when various bubbles collapse randomly with radiated noise waveform structures being different.

$$
|S(f)|^{2}=\left|\int_{0}^{\infty} \frac{\pi R}{2 R_{0}^{2}} e^{-\frac{\pi R^{2}}{4 R_{0}^{2}}} \int_{-\infty}^{\infty} P_{R}(f) e^{-j \omega t_{0}} d t_{0} d R\right|^{2}
$$




$$
=\left|\int_{-\infty}^{\infty} \int_{0}^{\infty} \frac{\pi R}{2 R_{0}^{2}} e^{-\frac{\pi R^{2}}{4 R_{0}^{2}}} P_{R}(f) d R e^{-j \omega t_{0}} d t_{0}\right|^{2}
$$

It is known from formula (13) that the smaller the degree of difference $\delta$ is, namely, the smaller the change of $P_{R}(f)$ is, the more similar the synthetic power spectrum is to base spectrum shape in terms of shapes when various cavitation bubbles waveform structures are different. To the contrary, the larger the shape change of synthetic power spectrum and base spectrum is, the smaller randomicity $\xi$ is, and the smoother the synthetic power spectrum is, otherwise, the synthetic power spectrum will be more rough..

The analysis above shows that Monte Carlo method could be used to carry out simulation modeling for propeller cavitation noise continuous spectrum waveform. The algorithm flow is indicated in Fig 4.

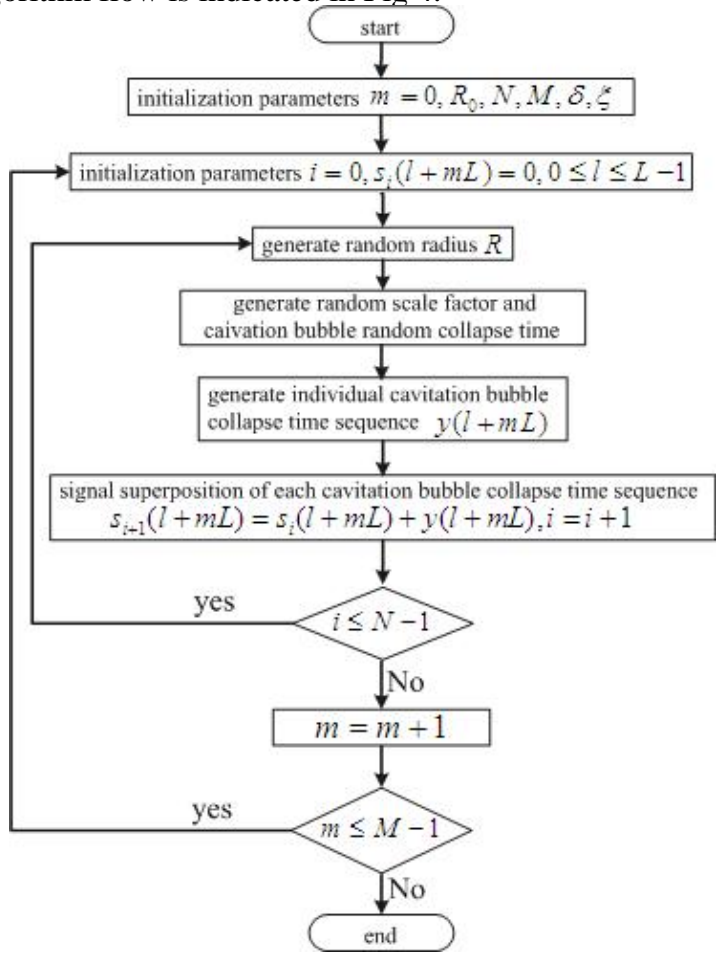

Figure 4. Algorithm flow chart of simulation model of cavitation noise continuous spectrum waveform

Wherein, $m$ is time sequence frame serial number, $R_{0}$ is average cavitation bubble radius, $N$ is number of cavitation bubbles, $M$ is number of simulation frames, $\delta, \xi$ respectively are degree of difference and randomicity parameter; $L$ is time sequence length and $R$ is Rayleigh Distribution random number, its expected value is $R_{0}$.

Fig 5 is simulation results when randomicity $\xi$ and degree of difference $\delta$ are given different values. The simulation results show that the smaller the degree of difference $\delta$, the more similar the synthetic power spectrum is to base power spectrum in terms of shapes, the smaller the randomicity is and the smoother the synthetic power spectrum is. This is matching theoretical analysis above.

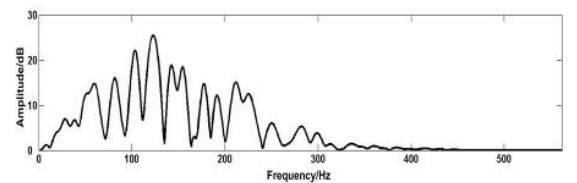

a.Synthetic power spectrum in case of $\boldsymbol{\xi}=100, \boldsymbol{\delta}=10$

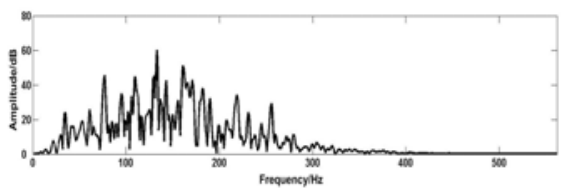

b.Synthetic power spectrum in case of $\xi=400, \delta=10$

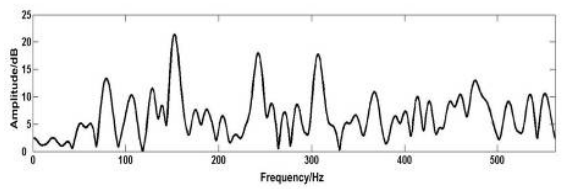

c.Synthetic power spectrum in case of $\xi=100, \delta=100$

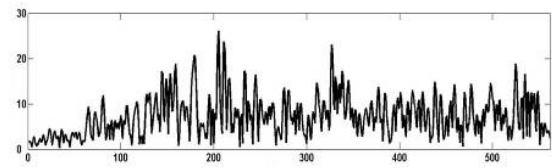

d.Synthetic power spectrum in case of $\xi=400, \delta=100$

Figure 5. Simulation results under different degrees of differences and different degrees of randomicity

The theoretical analysis and simulation results above show that the degree of difference $\delta$ can be used to control the similarity of shapes of synthetic power spectrum and base power spectrum and the degree of randomicity $\xi$ can be used to control smoothness of shape of synthetic power spectrum.

If cavitation bubble radiated noise waveform with expected radius being $R_{0}$ is used as base waveform and when collapse time $t_{0}$ of a large number of cavitation bubbles changes randomly, the broadband synthetic noise power spectrum could be worked out with formula (13) and in combination with degree of difference $\delta$ and degree of randomicity $\xi$. When input parameters are given, the results of formula (13) could be worked out through waveform statistics corresponding to different $R$ and $t_{0}$. Therefore, we call this model as time domain Monte Carlo simulation model. Fig 6 is comparison results between ship propeller cavitation noise continuous spectrum history simulated by this model and actual ship noise continuous spectrum history, 
which shows that this model has simulated the ship propeller cavitation noise continuous spectrum in a better way.

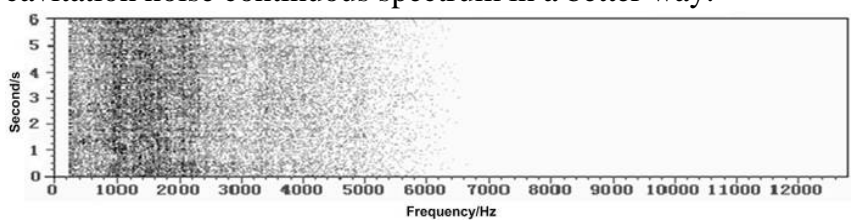

a. Simulation noise continuous spectrum history

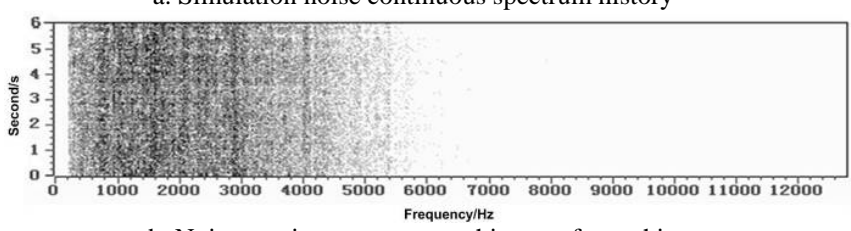

b. Noise continuous spectrum history of one ship

Figure 6. Comparison of simulation results and actual data

\section{CONCLUSION}

Based on theoretical research and test analysis of predecessors for cavitation noise continuous spectrum, time averaging treatment is made in this paper to the time variation of continuous spectrum caused by rotation of propeller. Theoretical analysis is made on synthetic power spectrum of each cavitation radiated noise in four circumstances according to collapse time of each cavitation bubble and its radiated noise waveform structure while propeller cavitation radiated noise continuous spectrum's expected value alone is taken into account. And also, corresponding simulation verification is made. The simulation result matches with theoretical analysis. Based on this, time domain Monte Carlo simulation model of cavitation noise continuous spectrum is proposed. The input parameters of this model include expected value of cavitation radius, degree of difference and degree of randomicity. The input parameters of this model are set up simply and have specific physical meanings. The simulation results show that this simulation model could simulate the cavitation noise continuous spectrum characteristics in a better way.

\section{ACKNOWLEDGMENT}

This research was supported by The National Basic Research Program (973 Program) (Grant No.613122103),the support is gratefully acknowledged.

\section{REFERENCES}

[1] Tao Duchun, "Propeller Cavitation Noise Spectrum," Acta Acustica.Beijing, vol.7, pp. 344 -350, June 1982.

[2] Jiang Guojian, Lin Jianheng, Ma Jie etc. "Mathematical Model of Ship Propeller Cavitation Bubble Noise, ” Acta Acustica.Beijing, vol.23, pp. 401-408, May 1998.

[3] P.T.Arveson,D.J.Vendittis. "Radiated noise characteristics of a modern cargo ship, ”J.Acoust.Soc.Am.NewYork,vol.107, pp. 118129, January 2000.

[4] P. Van Oossanen. "Theoretical Prediction of Cavitation on Propellers, ”Marine Technol. Soc. J. vol. NewYork, vol.14, pp. 391409, January 1997.

[5] D Rose, "Underwater Noise Principle, " Ocean Press. Beijing , pp. 70-71,1983.

[6] Deng Guoqiang, Sun Chao, Tang Jiansheng etc. "Time Domain Simulation of Typical Ship Radiated Noise, ”Torpedo Technology.Xi-an, vol. 13, pp. 41-43, April 2005.

[7] Liao Hongyu, "Modelling and Simulation of Passive Sonar Target / Background, ”Computer Simulation . Beijing, vol. 23, pp. 1-4, April 2006.

[8] Cheng Yijie, "Research on Simulation Technology of Ship Noise, ” China Technology Information. Beijing, vol. 19, pp. 273-274, February 2006.

[9] Zhang Bin, Sun Changyu, "Sea Test Data Analysis and Characteristics Simulation of Tow Boat Radiated Noise,” Acoustics Technology. Shanghai, vol. 26, pp. 798-801, May 2007.

[10] Bai Hanbin, Tian Peigen, Zhang Mingmin, "Research on Simulation Method of Ship Radiated Noise in One Sonar Simulation System Design, "Wuhan University of Technology Journal . Wuhan, vol.32, pp. 36-39, January 2008. 